\title{
Nuclear reaction models for systematic analysis of the fast neutron induced $(n, p)$ reaction cross sections
}

\author{
G. Khuukhenkhuu ${ }^{1, \text { a }}$, M. Odsuren ${ }^{1}$, B. Munkhbat ${ }^{1}$, Yu.M. Gledenov ${ }^{2}$, and M.V. Sedysheva ${ }^{2}$ \\ 1 Nuclear Research Center, National University of Mongolia, Ulaanbaatar, Mongolia \\ ${ }^{2}$ Frank Laboratory of Neutron Physics, JINR, Dubna, Russia
}

Abstract. Taking into account compound, pre-equilibrium and direct reaction mechanisms, we suggest certain method for theoretical explanation of systematic regularity in the fast neutron induced $(n, p)$ reaction cross sections. The statistical model, Griffin exciton model and PWBA are used. For systematical analysis of $(n, p)$ reaction cross sections, simple and convenient formulae are deduced. It is shown that theoretical $(n, p)$ cross sections are satisfactorily in agreement with experimental values for the $6,8,10,13,14.5$, and $16 \mathrm{MeV}$ neutrons.

\section{Introduction}

Investigation of charged particle emission reactions induced by fast neutrons is important for both nuclear reactor technology and the understanding of nuclear reaction mechanisms. In particular, the systematic study of $(n, p)$ reaction cross sections is necessary to estimate radiation damage due to hydrogen production, nuclear heating and transmutations in the structural materials of fission and fusion reactors. In addition, it is often necessary, in practice, to evaluate the neutron cross sections of the nuclides, for which no experimental data are available. Because of this, in last years we carried out the systematic analysis of known $(n, \alpha)$ and $(n, p)$ cross sections and observed so-called isotopic effect in the wide energy interval of neutrons for the broad mass range of target nuclei [1-3]. Also, some formulae for the systematic analysis of the fast neutron induced $(n, p)$ reaction cross sections using the statistical model, exciton model and PWBA were obtained [4-6]. In this paper the systematic analysis of known $(n, p)$ cross sections for fast neutrons using the theoretical model formulae is described.

\section{Theoretical models of nuclear reactions}

\subsection{Compound mechanism}

For fast neutrons, using the evaporation and constant nuclear temperature models, semi-classical approach for an inverse reaction cross section and $\Gamma \approx \Gamma_{n}$ approximation we can obtain the following general formula for the $(n, x)$ reaction cross section [4]:

$$
\sigma_{n, x}^{c o m}=\sigma_{c}(n) \frac{2 S_{x}+1}{2 S_{n}+1} \frac{M_{x}}{M_{n}} e^{\frac{Q_{n x}-V_{x}}{\Theta}}\left\{\frac{1-\frac{W_{n x}}{\Theta} e^{-\frac{W_{n x}}{\Theta}}-e^{-\frac{W_{n x}}{\Theta}}}{1-\frac{E_{n}}{\Theta} e^{-\frac{E_{n}}{\Theta}}-e^{-\frac{E_{n}}{\Theta}}}\right\} .
$$

Here $S_{x}, M_{x}$ (resp. $S_{n}, M_{n}$ ) are the spin and the mass of the particle $x$ (resp. neutron), $V_{x}$ the Coulomb potential for the particle $x ; Q_{n x}$ is the reaction energy; $\Theta=k T$ is the thermodynamic temperature; $W_{n x}=E_{n}+Q_{n x}-V_{x} ; E_{n}$ is the neutron

\footnotetext{
${ }^{a}$ Presenting author, e-mail: g_khuukhenkhuu@yahoo.com
}

energy. In equation (1) secondary particles and $\gamma$-emission are neglected. If we use the Weizsacker formula for the nuclear binding energy, the following expression for the (n, p) cross section can be written:

$$
\sigma_{n, p}^{c o m}=C \pi(R+\lambda)^{2} e^{-K \frac{N-Z+1}{A}},
$$

where

$$
C=\exp \frac{1}{\Theta}\left(\gamma \frac{2 Z-1}{A^{1 / 3}}+\frac{\Delta}{A^{3 / 4}}-V_{p}\right)
$$

and

$$
K=\frac{4 \xi}{\Theta} .
$$

Here $R, Z, N$ and $A$ are the radius, the proton and neutron numbers, and the mass number of the target nucleus, respectively; $\lambda$ is the wavelength of incident neutrons divided by $2 \pi$; $\Delta=\delta_{i}-\delta_{f} ; \xi, \gamma, \delta_{i}$ and $\delta_{f}$ are the constants of Weizsacker formula. The parameters $C$ and $K$ in equation (2) are usually determined by fitting to experimental cross sections. However, the parameters $C$ and $K$ can be obtained directly from equations (3) and (4). If we determine the nuclear thermodynamic temperature as in [7] and use the Fermi gas model [8], we get the following formula for the parameter $\mathrm{K}$

$$
K=4 \xi \sqrt{\frac{A}{13.5\left(E_{n}+Q_{n p}\right)}} .
$$

If we use the approximation $Z \gg 1$, for Coulomb potential of protons it is possible to get

$$
V_{p}=\frac{Z}{A^{1 / 3}} M e V .
$$

Thus, in the approximation $\Delta=0$ for the $(n, p)$ reaction the parameter $C$ is determined from equations (3) and (6) as follows:

$$
C=\exp \left\{Z A^{1 / 6} \frac{2 \gamma-1}{\sqrt{13.5\left(E_{n}+Q_{n p}\right)}}\right\} \text {. }
$$

So, from equation (5) and (7) it can be written that the parameters $C$ and $K$ are related as follows:

$$
\ln C=\frac{Z}{A^{1 / 3}}\left(\frac{2 \gamma-1}{4 \xi}\right) K
$$




\subsection{Pre-equilibrium mechanism}

In the framework of the pre-equilibrium nuclear reaction mechanism, using the Griffin exciton model we can write following expression for $(n, x)$ cross section [5]:

$$
\frac{d \sigma_{n, x}^{\text {pre }}}{d E_{x}}=\sigma_{r}\left(E_{n}\right) \sum_{n=n_{0}}^{n_{\max }} \lambda_{x}\left(n, E_{x}\right) \tau(n, E) .
$$

Here $\sigma_{r}\left(E_{n}\right)$ is the total reaction cross section for neutrons; $\lambda_{x}\left(n, E_{x}\right)$ is the emission rate from an $n$ exciton state $(n=p+h) ; p$ is the particle number; $h$ is the hole number. We assume the probability of nucleon emission from state with $n$ excitons $\gamma_{n} \approx 0$ and depleting factor $D \approx 1$. Also, for the transition probability is used the following approximation

$$
\lambda_{n}^{+} \gg \lambda_{n} \gg \lambda_{n}^{-}
$$

Then, the cross section formula for $(n, p)$ reaction can be obtained from equation (9) as follows

$$
\sigma_{n, p}^{p r e}=68.3 \frac{\pi^{6}}{\hbar^{2}} R^{2} \sigma_{r}\left(E_{n}\right) \frac{2 M_{p}}{K_{0} A} \frac{\left[\left(E_{n}+Q_{n p}\right)-V_{p}\right]^{3}}{\left(E_{n}+B_{n}\right)^{3}},
$$

where $M_{p}$ is the mass of protons; $K_{0} \approx 400 \mathrm{MeV}^{3}$.

\subsection{Direct reaction mechanism}

The transition probability of a system from the initial state to the final one is given by

$$
w=\frac{2 \pi}{\hbar}\left|\int \Psi_{f}^{*} H \Psi_{i} d \tau\right|^{2} \rho_{f}(E),
$$

where $H$ is the perturbation operator; $\rho_{f}(E)$ is the final states density; $\Psi_{i}$ and $\Psi_{f}$ are the wave functions for the initial and final states, respectively.

In the case of $(n, p)$ reaction we use following approximations for the initial and final states of the system [6]

$$
\Psi_{i}=U_{i} U_{n} \text { and } \Psi_{f}=U_{f} U_{p}
$$

where $U_{i}, U_{f}, U_{n}$ and $U_{p}$ are the wave functions of the target nucleus, product nucleus, neutron and proton, respectively. Also, we assume that the perturbation operator is equal to a constant

$$
H=C=\text { const. }
$$

The effect of the Coulomb field of nuclei is neglected as followings

$$
U_{f} \approx U_{i} \equiv U .
$$

In the case of plane wave Born approximation (PWBA) for neutrons and protons, from equations (12-15) we can write the following formula for the $(n, p)$ cross section

$$
\sigma_{n, p}^{d i r}=C_{0} \pi R^{2} \sqrt{1+\frac{Q_{n p}}{E_{n}}}
$$

Table 1. Energy dependence of the parameters $\mathrm{K}$ and $\mathrm{C}$.

\begin{tabular}{crr}
\hline $\mathrm{E}_{\mathrm{n}}(\mathrm{MeV})$ & $\mathrm{K}$ & $\mathrm{C}$ \\
\hline 6 & 75.2 & 17.5 \\
8 & 62.8 & 11.9 \\
10 & 52.1 & 6.80 \\
13 & 38.8 & 2.74 \\
14.5 & 37.3 & 2.43 \\
16 & 33.5 & 1.42 \\
\hline
\end{tabular}

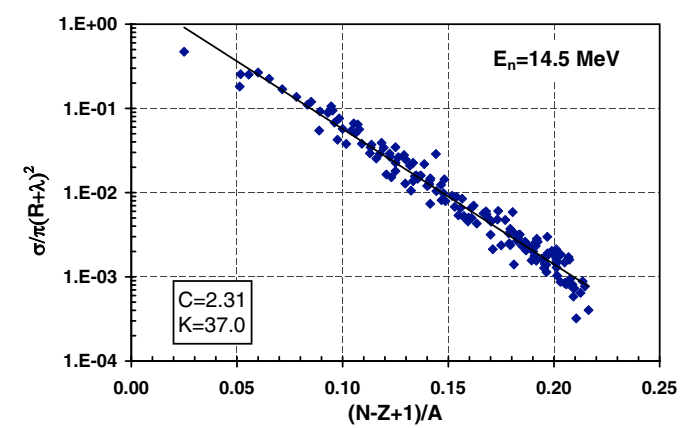

Fig. 1. Dependence of reduced (n, p) cross sections on the relative neutron excess parameter $(\mathrm{N}-\mathrm{Z}+1) / \mathrm{A}$ for neutron energy $14.5 \mathrm{MeV}$.

where $C_{0}$ is the fitting parameter which can be interpreted as a proton emission probability in the asymptotic total cross section for $E_{n} \gg Q_{n p}$.

So, the total $(n, p)$ cross section is determined as follows

$$
\sigma_{n p}=\sigma_{n p}^{c o m}+\sigma_{n p}^{p r e}+\sigma_{n p}^{d i r} .
$$

From equations (2), (11), (16) and (17) the parameter $C_{0}$ is obtained as best fit to experimental data.

\section{Analysis of $(n, p)$ cross sections}

\subsection{Systematics of $(n, p)$ cross sections}

Using equation (2) and fitting to experimental data we can determine the parameters $C$ and $K$ which are given in table 1 for $E_{n}=6,8,10,13,14.5$ and $16 \mathrm{MeV}$. The result of the fitting for $E_{n}=14.5 \mathrm{MeV}$ is shown in figure 1, as an example. It is seen that theoretical line fitted to experimental data is in agreement with known (n, p) cross sections. Similar results for other energy points were obtained.

The dependences of the parameters $K$ and $C$ on neutron energy are shown in figures 2 and 3, respectively. Figure 4 shows the dependence between the parameters $C$ and $K$ which were taken from the table 1 . From figures 2,3 and 4 it can be seen that the fitted values of parameters $C$ and $K$ are satisfactorily described by the statistical model formulae (5), (7) and (8).

Here the solid squares are the fits to the experimental data values (table 1) and the solid curves are the theoretical values. The values of $A, Z$ and $Q_{n p}$ are taken as averaged and constant parameters for all isotopes and given in figures 2 and 3, also. 


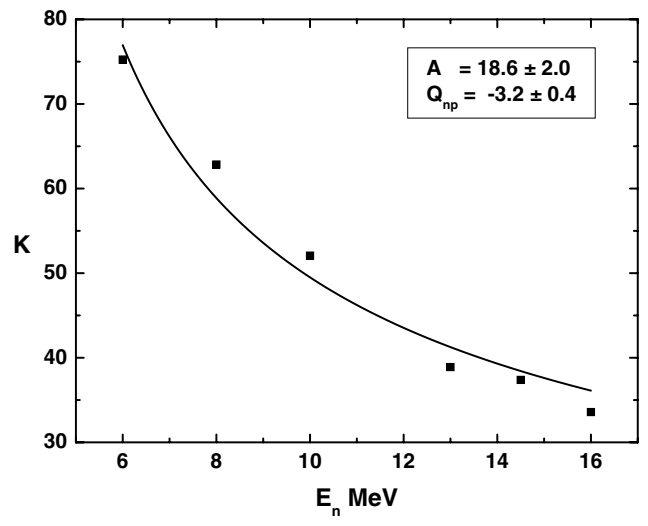

Fig. 2. Energy dependence of the parameter K.

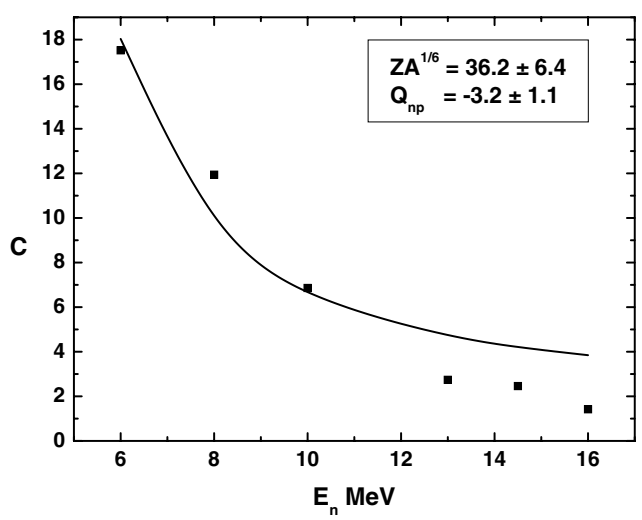

Fig. 3. Energy dependence of the parameter C.

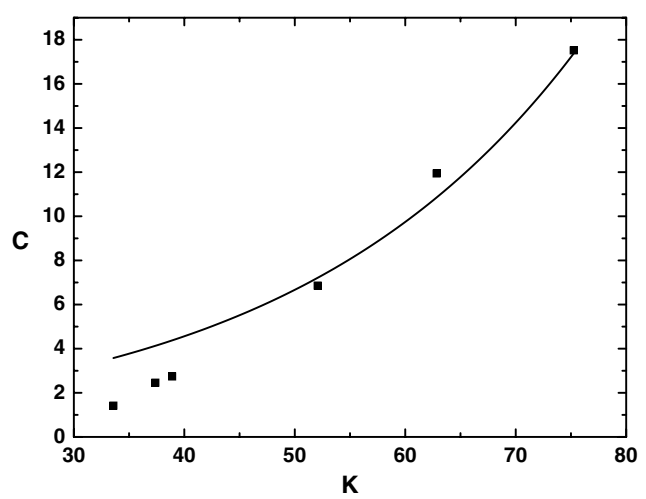

Fig. 4. The relationship between the parameters $\mathrm{C}$ and $\mathrm{K}$.

\subsection{Theoretical and experimental $(n, p)$ cross sections}

The comparisons of the absolute values for theoretical $(n, p)$ cross sections calculated by statistical model, exciton model and PWBA with known experimental data at $E_{n}=14.5 \mathrm{MeV}$ are shown, as an example, in figures 5, 6 and 7, respectively.

Also, in figures 8 and 9 are displayed the total $(n, p)$ cross sections calculated by equation (17) with experimental values for $E_{n}=8$ and $14.5 \mathrm{MeV}$. It is seen that the theoretical total $(n, p)$ cross sections are in agreement with experimental data. In the case of $E_{n}=6,10,13$ and $16 \mathrm{MeV}$ almost the same results were obtained.

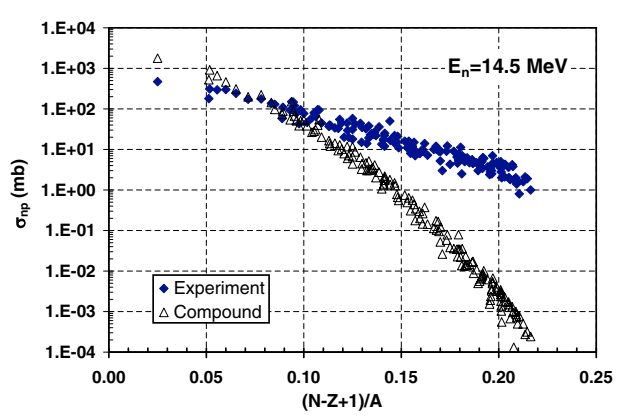

Fig. 5. Values calculated by the statistical model and experimental data of (n, p) cross sections at $14.5 \mathrm{MeV}$.

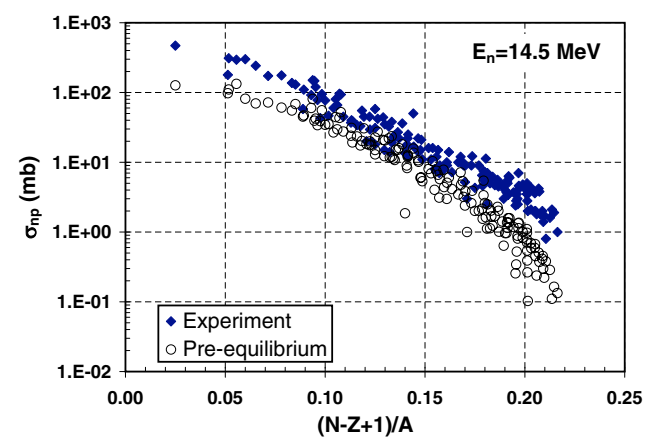

Fig. 6. Values calculated by the exciton model and experimental data of (n, p) cross sections at $14.5 \mathrm{MeV}$.

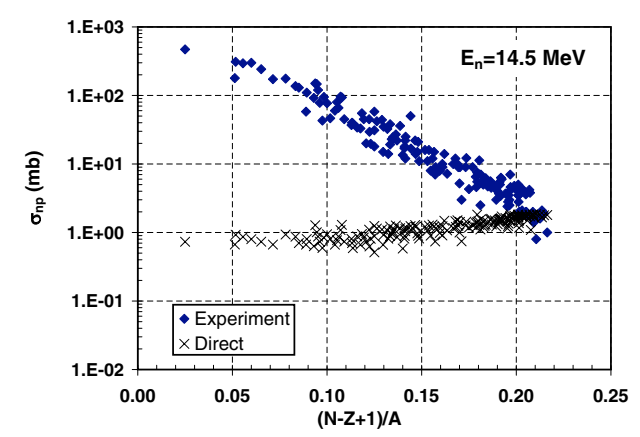

Fig. 7. Calculated by PWBA values and experimental data of (n, p) cross sections at $14.5 \mathrm{MeV}$.

\subsection{Analysis of the Levkovsky conclusions}

Levkovsky proposed three conclusions to explain the isotopic effect of $(n, \alpha)$ and $(n, p)$ cross sections for $E_{n}=14-$ $15 \mathrm{MeV}$ [9]. We revised these conclusions in the wide energy range of 6 to $16 \mathrm{MeV}$ and obtained following results:

1. Levkovsky empirical formula is almost identical with equation (2) which was deduced using the statistical model.

2. The ratio of the $(n, p)$ cross section to the $(n, \alpha)$ one is equal roughly to 2.5 for $E_{n}=8,10,13$ and $14.5 \mathrm{MeV}$, as concluded in [9]. But, at energies of $E_{n}=6$ and $16 \mathrm{MeV}$ this ratio is not equal to 2.5 . This effect, perhaps, is qualitatively explained by the difference of Coulomb barriers for protons and $\alpha$-particles in the same nucleus.

3. From our analysis it was seen that the systematical regularity of $(n, p)$ cross sections takes place not only at 


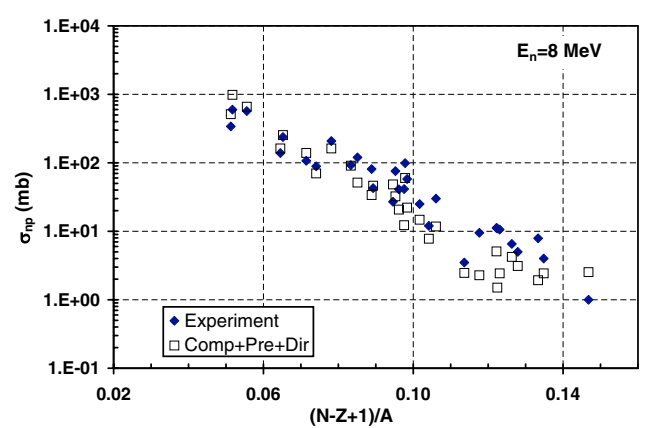

Fig. 8. Experimental and theoretical total (n, p) cross sections at $8 \mathrm{MeV}$.

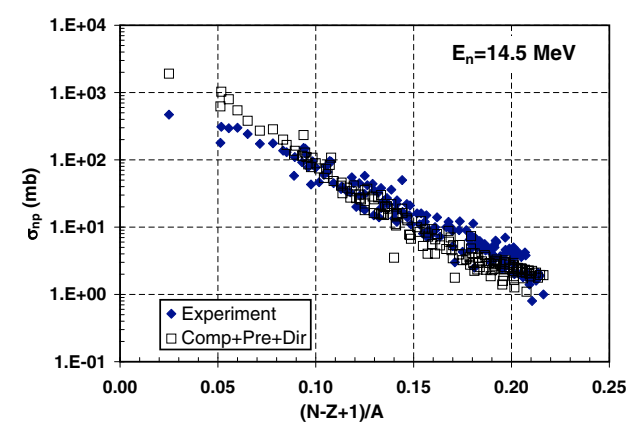

Fig. 9. Experimental and theoretical total (n, p) cross sections at $14.5 \mathrm{MeV}$.

14-15 MeV, as concluded in [9], but in the wide energy range of $E_{n}=6 \mathrm{MeV}$ to $16 \mathrm{MeV}$.
This work was carried out in the framework of the "Nuclear Reactions" project supported by Mongolian Science and Technology Foundation.

\section{References}

1. G. Khuukhenkhuu, Yu.M. Gledenov, M.V. Sedysheva, G. Unenbat, JINR Communications, E3-93-466, 1993, Dubna.

2. G. Khuukhenkhuu, Yu.M. Gledenov, M.V. Sedysheva, G. Unenbat, JINR communications, E3-94-316, JINR, 1994, Dubna.

3. G. Khuukhenkhuu, G. Unenbat, Yu.M. Gledenov, M.V. Sedysheva, in Proceedings of the International Conference on Nuclear Data for Science and Technology, Trieste, 1997, edited by G. Reffo, A. Ventura, C. Grandi, p. 934.

4. G. Khuukhenkhuu, G. Unenbat, Yu. Gledenov, M. Sedysheva, in Proceedings of International Conference on Nuclear Data for Science and Technology, Tsukuba, Japan, 2001, p. 782.

5. G. Khuukhenkhuu, Scientific Transactions, National University of Mongolia, 8(160) (2001), p. 21.

6. G. Khuukhenkhuu, in Proceedings of the 2-nd International School on Contemporary Physics, Ulaanbaatar, Mongolia, 9-19 September, 2002, edited by G. Khuukhenkhuu, p. 84.

7. J.M. Blatt, V.F. Weisskopf, Theoretical Nuclear Physics, (John Wiley and Sons, New York, 1952).

8. A. Bohr, B. Mottelson, Nuclear Structure (W.A. Benjamin, Inc., New York, Amsterdam, 1969).

9. V.N. Levkovsky, Yadernaya Fizika. 18(4) (1973), p. 705. 